\title{
H $\alpha$ photometry of low mass stars in 47 Tucanae: chromospheric activity and exotica
}

\author{
Giacomo Beccari ${ }^{1}$, Guido De Marchi ${ }^{2}$, Nino Panagia ${ }^{2,3,4}$ \\ \& Luca Pasquini ${ }^{1}$
}

\author{
${ }^{1}$ European Southern Observatory, Karl-Schwarzschild-Strasse 2, 85748 Garching bei München, \\ Germany, email: gbeccari@eso.org \\ ${ }^{2}$ ESA, Space Science Department, Keplerlaan 1, 2200 AG Noordwijk, The Netherlands \\ ${ }^{3}$ INAF-CT, Osservatorio Astrofisico di Catania, Via S. Sofia 78, 95123 Catania, Italy \\ ${ }^{4}$ Supernova Ltd, OYV \#131, Northsound Road, Virgin Gorda, British Virgin Islands
}

\begin{abstract}
We have used archival Hubble Space Telescope (HST) observations obtained with the Advanced Camera for Surveys (ACS) to study the $\mathrm{H} \alpha$ emission properties of main sequence (MS) stars in the globular cluster 47 Tucanae. An accurate photometric measurement of their $\mathrm{H} \alpha$ equivalent width, allows us to identify objects with large $\mathrm{H} \alpha$ emission. We demonstrate that this method allows us to identify and characterise candidate cataclysmic variables (CVs) with high efficiency, reconciling observations with theoretical predictions on the number of dynamically formed CVs expected in a 47 Tucanae-like globular cluster.
\end{abstract}

Keywords. Stars: chromospheres, cataclysmic variables, globular clusters: individual (47 Tucanae)

\section{Summary}

Using a method developed by De Marchi et al. (2010) we searched for stars showing $\mathrm{H} \alpha$ excess emission (Fig. 1) in the globular cluster 47 Tucanae. A proper combination of HST observations in the F606W (V), F814W (I) and F658N ( $\mathrm{H} \alpha$ ) bands, allows us to identify objects with large $\mathrm{H} \alpha$ emission and to perform an accurate photometric measurement of their $\mathrm{H} \alpha$ equivalent width $(\mathrm{EW}(\mathrm{H} \alpha)$; Fig. 1$)$, that we attribute to mass accretion rather than enhanced chromospheric activity. The spatial position of some of these stars is coincident with that of known X-ray sources and their location in the colour-magnitude diagram allows us to classify them as active binaries (ABs) or cataclysmic variables (CVs; Fig. 2). We demonstrate that this method allows us to identify and characterise for the first time a large number of candidate CVs in 47Tuc. In particular, we detect at least 12 candidate $\mathrm{CVs}$ in the region at $\mathrm{r}>70^{\prime \prime}$ from the cluster center, lying on the blue side of the cluster MS and showing an $\mathrm{H} \alpha$ excess emission with a measured $\mathrm{EW}(\mathrm{H} \alpha)>20 \AA$. Only 2 CVs were previously known from X-ray emission in the same region. Under the assumption that the ratio of $\mathrm{H} \alpha$ and $\mathrm{X}$-ray emitters is constant and of order 6 everywhere, we should expect to detect $\sim 90 \mathrm{CVs}$ with $\mathrm{H} \alpha$ emission inside the core radius, a number in full agreement with the theoretical expectations. Therefore, by allowing an efficient detection of candidate CVs, our method is able to reconcile theoretical expectation with observations. 


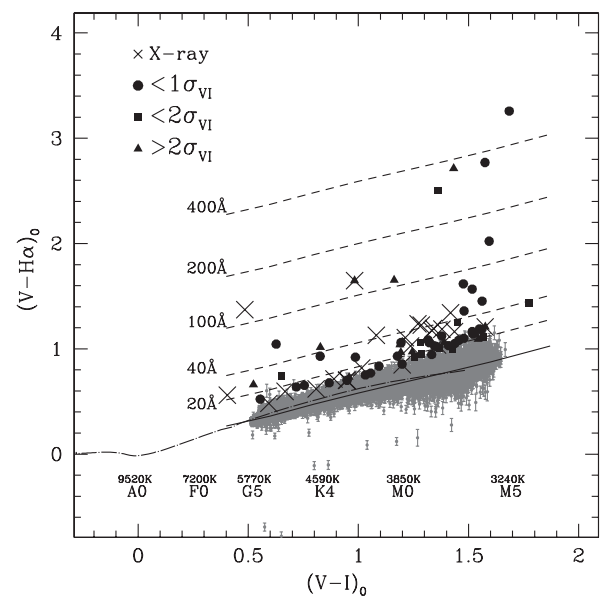

Figure 1. Selection of stars with $\mathrm{H} \alpha$ excess emission in a colour-colour diagram. The solid line represents the median $\mathrm{V}-\mathrm{H} \alpha$ colour representative of stars with no $\mathrm{H} \alpha$ excess and hence the location of stars with $\mathrm{EW}(\mathrm{H} \alpha)=0$. Dashed lines show the position of stars at increasing levels of $\mathrm{H} \alpha$ emission. All the objects with $\mathrm{H} \alpha$ excess emission at least 5 times larger than the photometric uncertainty on their $\mathrm{V}-\mathrm{H} \alpha$ colours are shown in black. X-ray sources showing $\mathrm{H} \alpha$ excess emission are shown as crosses, while solid circles, squares and triangles indicate $\mathrm{H} \alpha$ excess emitters lying on the CMD at $(\mathrm{V}-\mathrm{I})_{0}$ distance from the MS mean-ridge line, 1,2 or more than 2 times the combined photometric uncertainty on the colour. The dot-dashed line shows the location of the colour relationship derived for these bands using the Bessel et al. (1998) atmospheric models.

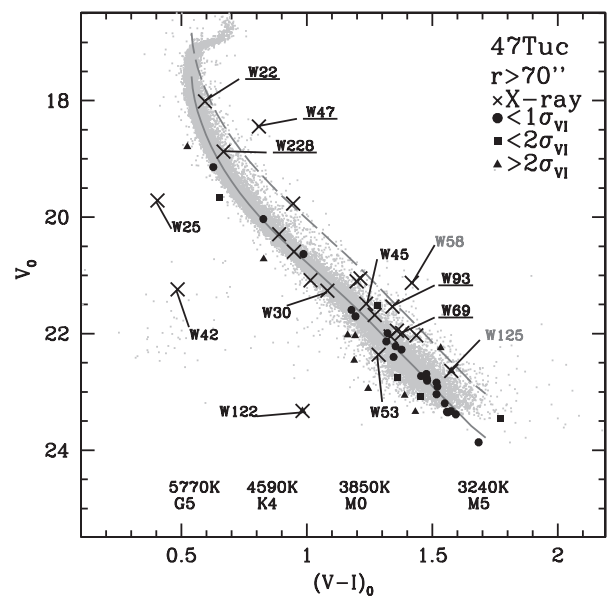

Figure 2. The stars with $\mathrm{H} \alpha$ excess emission and $\mathrm{EW}(\mathrm{H} \alpha>20 \AA)$ are shown as black symbols in the CMD of the cluster. Crosses mark the optical counterparts to X-ray sources across the whole field of view, whereas the grey data points and the $\mathrm{H} \alpha$ excess sources marked with solid circles, squares and triangles are located outside a $70^{\prime \prime}$ radius. The MS mean ridge line (grey solid line) and the spectral types are also shown. The 6 known CVs (black characters), the 5 ABs (underlined characters) and the 2 LMXBs (grey characters) are explicitly labeled.

\section{References}

Bessell, M. S., Castelli, F., \& Plez, B. 1998, A\&A, 333, 231

De Marchi, G., Panagia, N., \& Romaniello, M. 2010, ApJ, 715, 1 